\title{
XI. Jahresbericht des botanischen Tauschvereins in Wien im Jahre 1856.
}

Bis zu Ende des Jahres 1856 sind 276 Botaniker mit dem Vereine in Verbindung getreten. Von diesen haben sich 50 im Laufe des Jahres mittelst Einsendungen an demselben betheiligt, und es wurden von diesen im Ganzen über 31.000 Exemplare eingesendet, namentlich hat Herr:

Andorfer Alois, Pharmacie-Magister, in Langenlois. - Eingesendet 287 Expl. aus der Flora daselbst.

Bartsch Franz, in Wien. - Einges. 150 Expl. aus der Flora von Mähren.

Bayer Johann, in Win. - Einges. 449 Expl. aus der Flora von Wien und Ungarn.

Bilimek Dominik, Professor in Krakau. - Eing. 97 Expl, aus der Flora von Krakau.

Braun Dr. Professor, in Baireuth in Baiern. - Eing. 220 Expl. aus der Flora von Kärnthen und Tirol.

Braunstingel J., in Wels. - Eing. 1374 Expl. aus der Flora von Ober-Oesterreich.

Czetz Anton, in Ganis in Siebenbürgen. - Eing. 173 Expl. aus der Flora von Siebenbürgen.

Dollimer Dr. Georg, in Idria. - Eing. $700 \mathrm{Expl}$ aus der Flora von Krain.

Garcke Dr. August, in Berlin. - Einges. 355 Expl. aus der Flora von Preussen.

Hausmanu, Franz, Freiherr von, in Bolzen. - Einges. 301 Expl. aus der Flora von Tirol.

Heuser P., Lehrer in Gnadenberg in Schlesien. - Einges. 610 Expl. aus der Flora vou Schlesien und Westphalen.

Hillardt Christian, in Wien. - Einges. 2509 Expl, aus der Flora von Friaul.

Hölal J., Apotheker in Maria-Zell. - Eing. 200 Expl. aus der Flora von Steiermark.

Huter Rupert, in Brixen. - Einges. 682 Expl. aus der Flora von Tirol und ober-Italien.

Janka, Victor von, in Wien. - Einges. 690 Expl. aus der Flora von Siebenbürgen.

Jeehl Dr. Franz, Professor in Budweis. - Einges. 50 Expl. aus der Flora von Böhmen.

Juratzka Jac., k. k. Beamter in Wien. - Einges. 1440 Expl. aus der Flora von Wien.

Kintal Adolph, k. $k$ Hauptmann in Wiener - Neustadt. - Einges. 378 Expl. aus Nied.-Oesterreich.

Klinsmann, Dr. Ernst, in Danzig. - Einges. 60 Expl. aus der Flora daselbst.

Knebel, Wundarzt in Breslau. - Einges. 629 Exempl. aus der Flora von Schlesien. 
Kovatz Julius von, Custos in Pesth. - Eing. 160 Expl, aus der Flora von Ungarn.

Krabler Paul, in Achen. - Eing. 1000 Expl. aus der Flora von Ackien.

Krzisch Dr. Josef, Comitats-Phisicus in Tyrnau. - Eing. $265 \mathrm{Expl}$. aus der Flora von Ungarn.

Lagger Dr. Franz, in Freiburg in der Schweiz. - Eing. 1084 Expl. aus der Flora der Schweiz.

Makowsky Alexander, in Brünn. - Einges. 320 Expl. aus der Flora von Mähren.

Malinski Fr., Ingenieur in Bodenbach. - Einges. 282 Expl. aus der Flora von Böhmen.

Meyer J. L., Hof-Apotheker in Baireuth in Baiern. - Eing. $433 \mathrm{Expl}$. ans der Flora von Baiern.

Müller Ernst, in Ratibor. - Einges. $755 \mathrm{Expl}$. aus der Flora von Schlesien.

Müller Franz, Apotheker in Schneeberg in Sachsen. - Eing. 650 Expl, aus der Flora von Sachsen, Sardinien u. a.

Niessl Gustav von, in Wien. - Eing. 674 Expl. aus der Flora von Wien und Ungarn.

Oberleitner Franz, Cooperator in Neustift in Ober-Oesterreich.Einges. 837 Expl. aus der Flora von Ober-0esterreich.

Ortmann Johann, k. k. Beamter in Wien. - Eing. 266 Expl. aus der Flora von Wien.

Rauscher, Dr. Robert, in Wien. - Einges. 1253 Expl. aus der Flora von Wien und Oher-Oesterreich.

Rehm, Dr. H. in Dielenhofen, in Baiern. - Einges. 175 Expl. aus der Flora von Baiern.

Beichardt Heinrich, in Wien. - Eing. 617 Expl. aus der Flora von Mähren.

Roth Anton, Secretär in Prag. - Einges. 444 Expl. aus der Flora von Böhmen.

Schäde J., Cantor, in Alt-Reetz, in Preussen. - Einges. 351 Expl. aus der Flora von Preussen.

Scheidweiler, Professor in Gent. - Eing. 534 Expl, aus der Flora von Belgien.

Schlosser, Or. C., Comitats-Physicus in Agram. - Einges. $720 \mathrm{Expl}$. aus der Flora von Croatien.

Schmuek J. von, Pharmaceut in Brixen. - Eing. 169 Expl. aus der Flora von Tirol.

Schramm, Oekonomie-Rath in Brandenburg. - Eing. 170 Expl. aus der Flora von Preussen.

Schultz Dr. C. H., in Deidesheim - Eing. 700 Expl. aus der Flora von Baiern.

Schur Dr. Ferdinand, Professor in Wien. - Eing. 184 Expl, aus. der Flora von Siebenbürgen.

Serger Dr, in Seckenheim. - Eing. 500 Expl, aus der Flora von Baiern.

Siegmund Wilhelm, in Reichenberg. - Eing. 3900 Expl. aus der Flora von Böhmen. 
Stur Dionis, Reichs-Geologe in Wien. - Einges. 700 Expl. aus der Flora von Friaul und Istrien.

Uechtritz, R.v., in Breslau. - Einges. 1100 Expl. aus der Flora von Schlesien.

Val de Lièvre Anton, k. k. Finanz-Secretär in Innsbruck. Einges. 253 Expl. aus der Flora von Tirol.

Vukotinovic Lud. v., in Agram. - Einges. 545 Expl. aus der Flora von Croatien.

Waldmüller Fr., Pharm.-Magister in Botzen, - Einges. 580 Expl. aus der Flora von Tirol.

\section{Contimutio \\ Elemehi depplicatormen.}

Achillea cartilaginea $\mathrm{L}$ ed e b.

Aconitum ranunculifolium $\mathrm{R} \mathrm{ch}$. Allium ammophilum $\mathrm{He}$ a $\mathrm{f} \mathrm{f}$. Alnus autumnalis $\mathrm{Hart}$. Angelica montana S chlch. Anthemis tenuifolia S chur, Aster frutetorum $\mathrm{W}$ i m. Astrantia gracilis $\mathrm{B} \times \mathrm{t}$. Avena amethystina $\mathrm{Clar}$. Betula oycoviensis Bess. Biscutella ambigua D. C. Bulbocodium ruthenicum B ung. Bunium montanum $\mathrm{K}$ och. Bupleurum aureum $\mathrm{E}$ i s ch. polyphyllum L ede b. Calamagrostis pyramidalis $\mathrm{Hs} \mathrm{t}$. Calamintha Piperella $\mathrm{R} \mathrm{ch} \mathrm{b}$. Carex evoluta $\mathrm{H}$ a $\mathrm{r} \mathrm{m}$. "orthostachis C. A. M. " strigosa All.

Cerastium ciliatum W. K. Chrysocoma villosa $\mathrm{L}$.

Crocus Heuffelianus $\mathrm{H}$ e $\mathrm{r} \mathrm{b}$. Cucumis Melo L.

Dianthus gramineus Schur.

Dichostilis fluitans P. B.

Diospyros Lotus $\mathrm{L}$.

Echinophora spinosa L.

Euphorbia incana $\mathrm{S}$ ch u r.

Heracleum siifolium $\mathrm{R} \mathrm{chb}$.

Herminium clandestinum $\mathrm{Gr}$ en. Hieracium caesium var. macula-

$$
\begin{aligned}
& \text { tum } \mathrm{Schlt} \mathrm{z} . \\
& \text { prenanthoidi-murorum }
\end{aligned}
$$$$
\text { 9. }
$$$$
\text { Sch Itz. }
$$

Hypericum elegans $\mathrm{S}$ te $\mathrm{p} h$.
Iris ruthenica A it.

Koeleria grandiflora R. el Sch. Myriophyllum alterniflorum D. C. Nigrilella fragrans $\mathbf{R} \mathbf{c} \mathbf{h}$.

Onobrychis saxatilis All. Orobanche Picridis Schltz. Orobus versicolor Gmel.

Phlomis tuberosa L.

Pimpinella Anisum L.

Potentilla frigida $\mathrm{V} \mathrm{i} l \mathrm{l}$.

Ranunculus pedalus $\mathbf{W}$. K.

Rhododendron myrtifolium $\mathrm{Sch} \mathrm{t}$. Rubus Köhleri W. K.

$"$ thyrsiflorus $\mathrm{W}$ h.e.

" vulgaris $W$ he.

Ruppia rostellata $\mathrm{Koch}$.

Salix incubacea W11d.

Saponaria bellidifolia $\mathrm{S} \mathrm{m}$.

Saxifraga hypnoides L.

Schoberia salsa M a y.

Scilla bifolia var. ruthenica J a $\mathrm{n} k$.

Sempervivum glaciale Braun.

Seneoillis carpathica $\mathrm{Sch}$ ot t.

Seseli gracile W. K.

Sesleria marginata Gri es b.

Silene pusilla W. K.

" Siegeri B m g.

"Zawadskii He r b.

Sorghum vulgare P e r s.

Stachys obliqua W. K.

Syringa Josikaea J c q.

Tamarix gallica $\mathrm{L}$.

Teucrium euganeum $\mathrm{V}$ is.

supinum $\mathbf{J} \mathrm{eq}$.

Thalictrum peucedanifolium

Gries b. 
Thlaspi calaminare L $\mathrm{L} \mathrm{j}$. Thymus comosus He uff. Udora occidentalis P r s c h. Vallerianella pumila D. C. Verbascum sinuatum L a m. Veronica saturioides Vis.

Viola multicaulis $\mathrm{J}$ or d. " permixia Jord. \# scotophylla J ord. " suavissima Jord. "Valderia All.

Waldsteinia trifolia $\mathrm{R}$ o $\mathrm{c}$ h el.

\section{Kryptogamen.}

(Nomenclatur nach Dr. L. R a b e $\mathbf{n}$ h o rs t.)

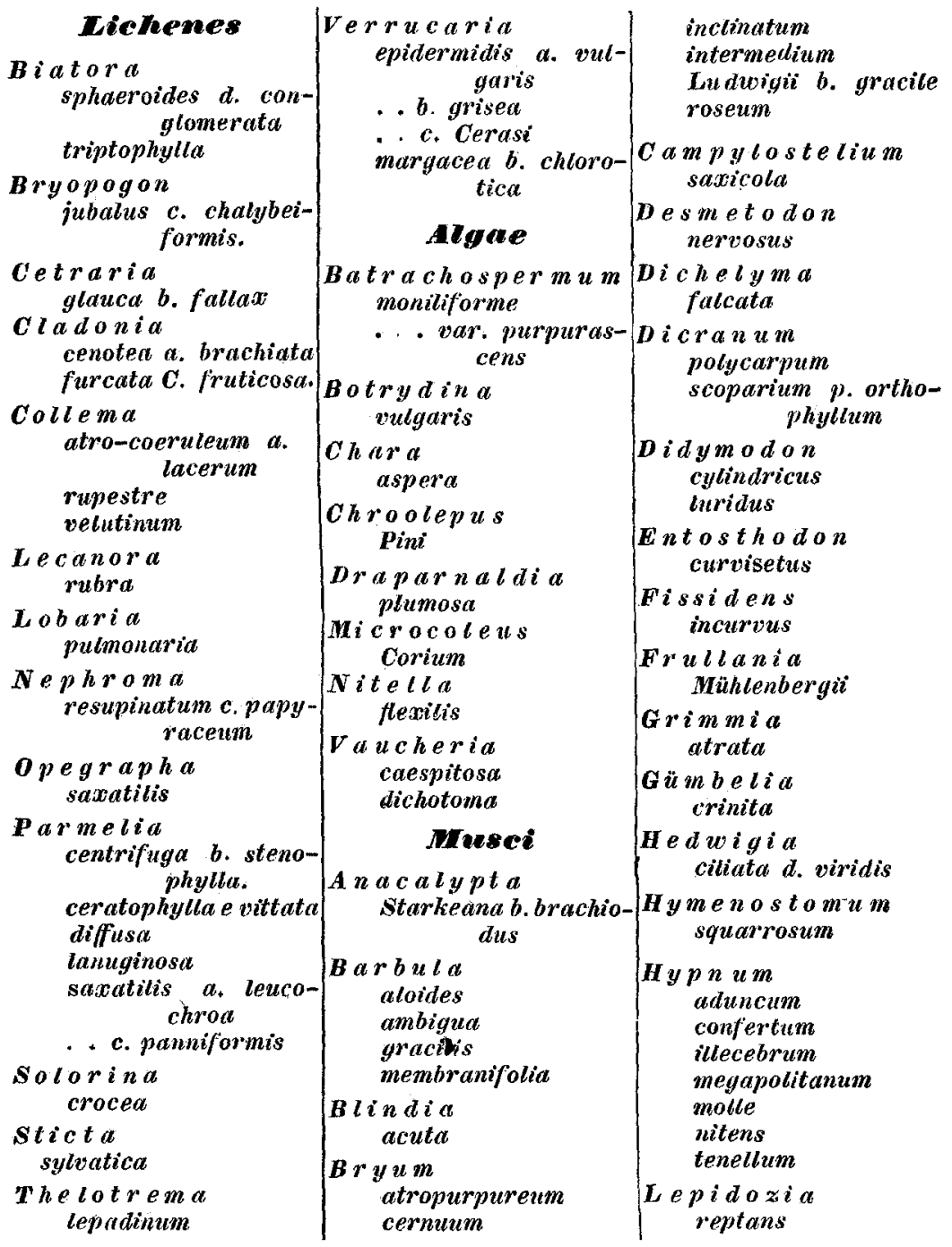




\begin{tabular}{|c|c|c|}
\hline & 60 & \\
\hline 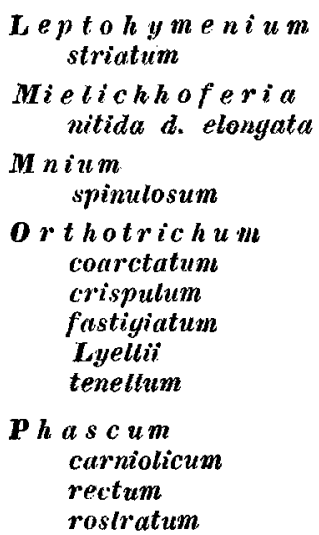 & 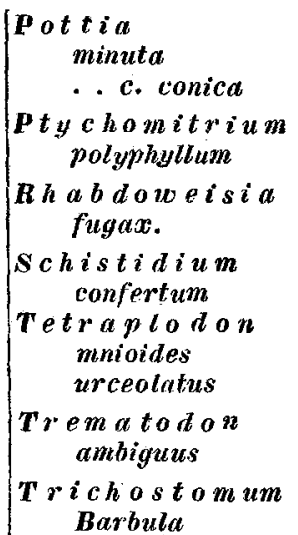 & 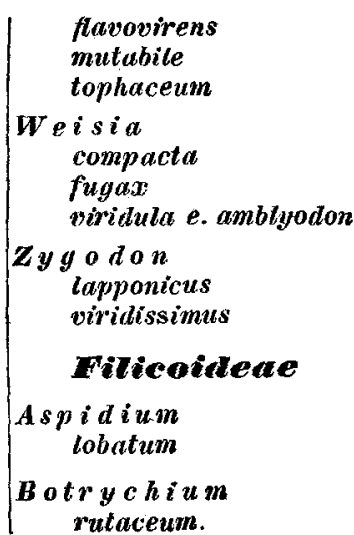 \\
\hline
\end{tabular}

Wien, (Wieden Nr. 331) 1. Jänner $185 \%$.

Dr. Alexander Skofitz.

\section{Mittheilung.}

- Im $S$ a menbau und $S$ a m en hande I wird Erfurt noch durch Quedlinburg, Aschersleben und Eisleben weit ubertroffen. Eine einzige Handlung dortselbst verkauft jährlich allein über 1000 Centner Zwiebelsamen, der mit andern Sachen zum Theil daselbst nach Amerika und anderen Weltheilen geht. Ueber $\mathbf{8 0 0}$ Morgen verwendet diese zu ihrem Anhau der verschiedenen Samereien und bezieht ansserdem noch sehrviel von den kleineren Grundbesitzern. Eine zweite Gärtnerei bestellt jährlich 600 Morgen, während noch andere 150-200 Morgen zur Samengewinnung benützen. Man sieht oft eiı Stück Landes von 3-4 Morgen nur besäet mit Asterll. Es versteht sich übrigens von selbst, dass auch neben Blumen- und Gemüse-Samen allerhand ökonomische Sämereien gezogen werden.

\section{I $\mathbf{s}$ e a t e.}

\section{Anzeige von Gemüse-, Feld-, Gras-, Blumen-, Oekonomie- und Wald-Samen, Pflanzen und Knollen.}

Der Unterzeichnete erlaubt sich hierdureh darauf aufmerksam za machen, dass sein $n$ e u s sehr relehhanciges Verzeichniss für 1857 über obige Artikel erschienen, und auf lrankirtes Verlangen, sowohl durch Unterzeichneter, als anch bei flerrn will h. Sabimsixy in Wiem, Stadt Nr. 654, welcher nuch Bestellungen annimmt, gratis za baben ist

Die Preise sind für vor ü̈gliche, zuverlässig ech be ke imfäh ige Sa a t möglichst billig gestellt. Alle Aufträge werden, wie seit vielen Jahren, prompt und reell zur $Z$ ufriedenheit der Herren Auftraggeber ausgeführt.

E r f u r, im Jänner 1857.

Ernst Benary,

Samenhabdlang, Kunst- und Handelsgärtnerei.

Der heutigen Nımmer liegt bei : „Preis-Courant der Samen- und Planzenhandlung der Gebrüder Vill a i n in" Erfurt."

Redacteur und Herausgeber Dr. Alexander Skofitz.

Verlug von L. W. S e id el. Druek von C. U e ber re uter. 